\title{
Asymptomatic rheumatic heart disease in South African schoolchildren: Implications for addressing chronic health conditions through a school health service
}

\author{
M Shung-King, ${ }^{1}$ DPhil; L Zühlke, ${ }^{2,3} \mathrm{PhD}$; M E Engel, ${ }^{2} \mathrm{PhD} ; \mathbf{B}$ M Mayosi, ${ }^{2} \mathrm{DPhil}$ \\ ${ }^{1}$ Health Policy and Systems Division, School of Public Health and Family Medicine, Faculty of Health Sciences, University of Cape Town, \\ South Africa \\ ${ }^{2}$ Department of Medicine, Groote Schuur Hospital and Faculty of Health Sciences, University of Cape Town, South Africa \\ ${ }^{3}$ Department of Paediatrics and Child Health, Red Cross War Memorial Children's Hospital and Faculty of Health Sciences, University of \\ Cape Town, South Africa
}

Corresponding author: M Shung-King (maylene.shungking@uct.ac.za)

\begin{abstract}
When new evidence comes to light, it compels us to contemplate the implications of such evidence for health policy and practice. This article examines recent research evidence on the prevalence of asymptomatic rheumatic heart disease (RHD) in South Africa and considers the implications for the Integrated School Health Programme (ISHP). RHD is still a major burden of disease in developing countries, and elimination of this preventable condition ranks high among World Heart Federation goals. If left untreated, it becomes a chronic health condition that individuals have to cope with into their adult lives. The ISHP regards the health needs of children with chronic health conditions, which include conditions such as RHD, as a key service component. However, the chronic health component of the ISHP is still poorly developed and can benefit from good evidence to guide implementation. A recent study to ascertain the prevalence of RHD in asymptomatic schoolchildren through mass screening affords an opportunity to reflect on whether, and how, asymptomatic chronic health conditions in schoolchildren could be addressed, and what the implications would be if this were done through a school-based programme such as the ISHP.
\end{abstract}

S Afr Med J 2016;106(8):761-762. DOI:10.7196/SAMJ.2016.v106i8.10756

Strategies on how to address the health needs of children with chronic health conditions are increasingly receiving international attention as the global focus on non-communicable diseases escalates. ${ }^{[1]} \mathrm{A}$ recent study on the prevalence of rheumatic heart disease (RHD) in asymptomatic children, conducted in South Africa (SA) and Ethiopia, ${ }^{[2]}$ presents interesting lessons for conceptualising and addressing the needs of children with chronic health conditions through a school health programme such as the Integrated School Health Programme (ISHP) in SA. ${ }^{[3]}$

An important component of the wide range of health needs that the ISHP in SA aims to address is the identification and support of children with chronic health conditions throughout their school years. However, the ISHP provides little practical guidance on how this should be done. ${ }^{[4,5]}$ The findings of a study identifying the presence of RHD in a cohort of asymptomatic and undiagnosed schoolchildren present an opportunity to contemplate what these findings mean for addressing the needs of schoolgoing children with chronic health conditions in the context of the ISHP.

\section{Study findings and implications}

The study established the prevalence of RHD by screening randomly selected schoolchildren from 4 through to 24 years of age. ${ }^{[2]}$ It employed the standardised echocardiographic diagnostic criteria of the World Heart Federation (WHF) ${ }^{[6]}$ The study spanned two study settings: the Bonteheuwel and Langa communities of Cape Town, SA, and Jimma, Ethiopia. The varying socioeconomic gradient across the study sites increased the applicability of the findings to a range of socioeconomic contexts.
A total of 2720 schoolchildren (mean age 12.2 (standard deviation (SD) 4.2) years) were screened in SA, of whom the greater proportion $(58.9 \%)$ were female. In Ethiopia $(N=2000)$ the sex distribution was equal, with a younger mean age of 10.7 (SD 2.5) years. The mean prevalence in the SA arm of the study was $20.2 / 1000$, with the prevalence of 27/1 000 in Langa, double the 12.5/1 000 in Bonteheuwel, demonstrating an important socioeconomic gradient, even in two geographically contiguous urban communities. The even higher prevalence of 30.5/1 000 in Jimma, Ethiopia, where circumstances are more akin to those in rural SA, suggests that a similar higher prevalence can be expected in poorer rural areas. Key study conclusions are summarised in Table 1.

Two subsequent questions that emerged for the study team were: (i) whether some children may have left school as a result of symptoms related to RHD, which would be picked up only through a community-based study; and (ii) the extent to which asymptomatic children who remain undiagnosed continue into adulthood with good health and no impact on their schooling. The study team also recognised the controversies that exist around: (i) the method of screening for asymptomatic RHD; (ii) the borderline disease entity; and (iii) the prognostic impact of asymptomatic RHD. ${ }^{[8]}$ The WHF criteria, which represent the only evidence-based standardised guideline for the diagnosis of asymptomatic RHD and have been applied to both high- and low-risk populations, ${ }^{[9]}$ are not without criticisms, particularly with regard to their high specificity and low sensitivity. ${ }^{[10]}$ The practical implications for longer-term screening are still unclear. ${ }^{[11]}$ Recent publications of long-term follow-up have divergent recommendations for repeat screening in endemic populations, ${ }^{[10]}$ including those in which previous 


\section{Table 1. Key study findings ${ }^{[2]}$}

1. A high prevalence of RHD in asymptomatic schoolchildren. The prevalence of echocardiographic RHD of 20.2/1 000 in Cape Town was much higher than the 6.9/1 000 previously estimated using clinical auscultation over 30 years ago, ${ }^{[7]}$ meaning that the new standardised echocardiographic method is more sensitive and likely to identify larger numbers of undetected children than previous techniques.

2. The differing odds of detecting echocardiographic RHD between countries and between adjacent communities in SA points to the pronounced gradient across differential socioeconomic conditions, with increasing prevalence occurring with increasing levels of poverty

3. This socioeconomic gradient between countries and communities also manifested in the severity of asymptomatic RHD. Schoolchildren from Ethiopia had the more severe variant, followed by Langa and then Bonteheuwel.

4. Based on these findings, there appears to be a substantial pool of schoolchildren in SA and Ethiopia with definite and borderline RHD that remains undetected. How to respond to these findings in the context of a school health programme requires consideration.

screening was negative, as well as for follow-up at regular intervals (including borderline disease) to monitor clinical progression. ${ }^{[12]}$ However, pending more definitive studies of impact on prognosis, echocardiographic screening for asymptomatic RHD remains, for now, more of a research tool. ${ }^{[13]}$

\section{Some key lessons and considerations}

The study convincingly showed that the detection of asymptomatic disease requires a screening process and tools that are effective and easy to administer, with high sensitivity and specificity, to appropriately identify children with the condition of interest. Does the availability of such a screening process for RHD merit its automatic inclusion into the mass screening programme of the ISHP? Recent debates on the implementation of the current ISHP point to the need to reduce, rather than increase, mass screening. ${ }^{[4,5]}$ In these debates, task-shifting of mass screening activities to a well-trained lower cadre of health workers is further proposed, in contrast to the method used in the study, where professional echocardiographers were required to do the screening. This points to a need for careful contemplation of the implementation context, despite meritorious evidence. Further considerations from the study, that are in line with international screening criteria considerations and have implications for similar chronic health conditions, are: (i) the large numbers of screens that are required to identify a small number of cases; (ii) the unclear longterm prognosis of asymptomatic and borderline RHD in children; and (iii) the current capacity of health services to respond adequately to children who may need further testing, follow-up or surgery. ${ }^{[14,15]}$

In a school setting, children with chronic health conditions fall into one of the following main categories: (i) asymptomatic children, such as in this study; (ii) symptomatic children who are as yet undiagnosed; and (iii) children with confirmed diagnoses, who may be on treatment but are not identified in the school and school health service systems as needing support. This study prompts us to consider how to approach children who fall within these categories. As already indicated, mass screening to detect disease in asymptomatic children is not necessarily a viable option for RHD or for any other chronic health condition. An alternative is to focus targeted screening on other easily accessible asymptomatic groups at risk of complications, such as adolescent girls who attend antenatal services - this merits further debate.

Children who are symptomatic, and in particular those who have symptoms severe enough to impact on their general wellbeing and schooling, urgently require mechanisms to identify and fully support them. Equally important are considerations of how to follow up and monitor children with early, as yet uncomplicated conditions, where early identification can prevent progression and complications. It is here that the ISHP in partnership with schools can play a crucial role. Current research into potential models of early identification and support for children with mental health conditions is in progress, and may help in informing a broader service model covering all children with chronic health conditions.

For RHD, improvement in socioeconomic circumstances is the core to eradication. Beyond this, having strong and effective primary healthcare services is essential, where all children who require antibiotic treatment for suspected streptococcal sore throat are appropriately treated and followed up, and where secondary prevention measures are available. ${ }^{[16]} \mathrm{A}$ well-functioning ISHP has a crucial health promotion and ongoing monitoring and support role. Simply raising awareness among educators and parents of the need to treat sore throats is an important initial intervention that could lead to greater vigilance and care of children. ${ }^{[17,18]}$ While policy makers and practitioners grapple with how to appropriately respond to the now confirmed population of children with asymptomatic RHD, and the yet unknown population of children with other undiagnosed chronic health conditions, there is an opportunity to equip educators, parents and caregivers with simple guidelines on how to identify children who display signs of difficulty, whether physical or mental, while working towards strengthening the ISHP and school system capacity. 2012;130(3):379-381. DOI:10.1542/peds.2012-1475

2. Engel ME, Haileamlak A,Zuhlke L, et al. Prevalence of rheumatic heart disease in 4720 asymptomatic scholars from South Africa and Ethiopia. Heart 2015;101(17):1389-1394. DOI:10.1136/heartinl-2015-307444

3. National Department of Health, South Africa. Integrated School Health Policy. Pretoria: NDoH, 2012.

4. Shung-King M. From 'stepchild of primary healthcare' to priority programme: Lessons for the Shung-King M. From stepchild of primary healthcare to priority programme: Lessons for the
implementation of the National Integrated School Health Policy in South Africa. S Afr Med J implementation of the National Integrated Sch

5. Shung-King M, Orgill M, Slemming W. School health in South Africa: Reflections on the past and

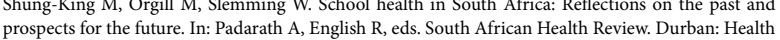
prospects for the future. In:
Systems Trust, 2014:59-72.

6. Reményi B, Wilson N, Steer A, et al. World Heart Federation criteria for echocardiographic diagnosis of rheumatic heart disease - an evidence-based guideline. Nat Rev Cardiol 2012;9(5):297-309. DOI:10.1038/nrcardio.2012.7

7. McLaren MJ, Hawkins DM, Koornhof HJ, et al. Epidemiology of rheumatic heart disease in black schoolchildren of Soweto, Johannesburg. BMJ 1975;3(3755):474-478.

8. Roberts K, Colquhoun S, Steer A, Remenyi B, Carapetis J. Screening for rheumatic heart disease: Current approaches and controversies. Nat Rev Cardiol 2013;10(1):49-58. DOI:10.1038/nrcardio.2012.157

9. Roberts K, Maguire G, Brown A, et al. Echocardiographic screening for rheumatic heart disease in high and low risk Australian children. Circulation 2014;129(19):1953-1961. DOI:10.1161/ CIRCULATIONAHA.113.003495

10. Tani LY. Echocardiographic screening for rheumatic heart disease. Circulation 2014;129(19):19121913. DOI:10.1161/CIRCULATIONAHA.114.009406

11. Zühlke L, Engel ME, Lemmer CE, et al. The natural history of latent rheumatic heart disease in a 5 year follow-up study: A prospective observational study. BMC Cardiovasc Disord 2016;16(1):46.
D 5 year follow-up study: A prospe
DOI:10.1186/s12872-016-0225-3.

12. Mirabel M, Fauchier T, Bacquelin R, et al. Echocardiography screening to detect rheumatic heart disease: A cohort study of schoolchildren in French Pacific Islands. Int J Cardiol 2015;188:89-95. DOI:10.1016/j.ijcard.2015.04.007

13. Zühlke L, Mayosi BM. Echocardiographic screening for subclinical rheumatic heart disease remains a research tool pending studies of impact on prognosis. Curr Cardiol Rep 2013;15(3):343. DOI:10.1007/ s11886-012-0343-1

14. Andermann A, Blancquaert I, Beauchamp S, Déry V. Revisiting Wilson and Jungner in the genomic age: A review of screening criteria over the past 40 years. Bull World Health Organ 2008;86(4):317-319. 15. Wilson J, Jungner G. Principles and practice of screening for disease. Arch Intern Med 1969;123(3):349. 16. Irlam JH, Mayosi BM, Engel ME, Gaziano TA. A cost-effective strategy for primary prevention of acute rheumatic fever and rheumatic heart disease in children with pharyngitis. S Afr Med J 2013;103(12):894-895. DOI:10.7196/SAMJ.7244

17. Nordet P, Lopez R, Duenas A, Sarmiento L. Prevention and control of rheumatic fever and rheumatic heart disease: The Cuban experience (1986-1996-2002). Cardiovasc J Afr 2008;19(3):135-140.

18. Robertson KA, Volmink JA, Mayosi BM. Towards a uniform plan for the control of rheumatic fever Robertson KA, Volmink JA, Mayosi BM. Towards a uniform plan for the control of rheumatic fever
and rheumatic heart disease in Africa - the Awareness Surveillance Advocacy Prevention (A.S.A.P.) and rheumatic heart disease in Africa - the Awr
Programme. S Afr Med J 2006;96(3):241-245.

Accepted 16 March 2016. 